\title{
DISCUSSION: Rotation and Stellar Evolution
}

\author{
Ian W Roxburgh, Chairman \\ Astronomy Unit, Queen Mary, University of London, Mile End Road, \\ London E1 4NS, UK
}

\section{Introduction}

During the course of the meeting several of you have been kind enough to refer to some of my early work on stellar rotation. But the references have been selective; my early work was not on stellar rotation alone but on rotation and magnetic fields; my PhD thesis was entitled The Theory of Rotating Magnetic Stars and my early papers were on the generation of magnetic fields and circulation in rotating magnetic stars. I want to stress that

\section{Magnetic fields are not optional - they exist in nature.}

Magnetic fields are present in the interstellar matter out of which stars are formed so we should expect that stars contain a "fossil" field when they are first formed. Even if no magnetic field were present initially then a field would be generated by the "battery" effect of the electron pressure gradient in a differentially rotating star. Magnetic fields can be enhanced by dynamo action in both stellar cores and envelopes, differential rotation leading to a very substantial growth in the field and the dynamo cycle being closed by convective motions in stellar convective envelopes and in convective cores, or by magnetic instabilities.

Traditionally we have thought of dynamos as operating in convective regions but, as shown by $\mathrm{H}$ Spruit, dynamo action may also take place in radiatively stable regions of differentially rotating stars. Given a weak initial poloidal field differential rotation winds up the field producing a strong toroidal field. When the toroidal field is large enough it becomes unstable to a Tayler instability which generates poloidal field from the toroidal field. The resulting field is found to be more effective in transporting angular momentum than rotationally driven hydrodynamic instabilities. Chemical composition gradients are stabilising and can reduce the transfer of angular momentum.

$\mathrm{S}$ Brun showed some new results on simulations of turbulent convection and magnetic field enhancement in convective cores, although these simulations are not yet developed to the stage where the system is thermally relaxed. $\mathrm{He}$ emphasised that a magnetic field can both enhance and suppress different processes and $\mathrm{H}$ Spruit emphasised that small scale motions/instabilities can be very seriously modified by a magnetic field. 
Magnetic fields can be very effective at transporting angular momentum and thus controlling the evolution of the rotation inside a star, and hence any mixing caused by rotationally driven circulation or hydrodynamic instabilities.

If we ignore magnetic fields in our investigations into the effect of rotation on stellar evolution then we must explain how and why there is no magnetic field, or why its effect can be neglected in our investigation.

\section{Some Questions}

Let me pose a set of questions to which I would like to hear some answers:

We have heard about mixing processes driven by rotation but how is this affected by magnetic fields (and by composition gradients).

$\mathrm{P}$ Garaud reminded us that she has studied the problem of the evolution of the rotation field and circulation in a rotating magnetised stars and found that a magnetic field localised the circulation pattern. This in turn may lead to highly non-symmetric mixing. In the chairman's view the effect of magnetic fields on the rotationally driven instabilities which help to redistribute angular momentum needs detailed investigation as does the the effect of chemical composition gradients on both circulation and instabilities.

Can different regions of a star become magnetically uncoupled - with the core spinning faster than the envelope?

As we heard from J Christensen-Dalsgaard, in the only example we have of the internal rotation of a star, namely the internal rotation of the Sun deduced from helioseismology, the rotation of the radiative core is consistent with uniform rotation of magnitude equal to that at mid latitudes of the base of the solar convective envelope. On the other hand S Wolff, S Strom and L Hillenbrand argue that the surface convective zone in A type stars is effectively uncoupled from the radiative interior so the core remains more rapidly rotating than the convective envelope, hence explaining the drop in observed $v \sin i$ at spectral type A. H Langer pointed out that neutron stars are example of fast rotating stars with a magnetic field - and the core must have decoupled from the outer layers of the star prior formation of the neutron star. A Maeder argued that to explain the evolution of $\mathrm{O}$ and $\mathrm{B}$ stars the envelopes must be uncoupled from the core and that the Sun is in a different parameter range from these early type stars.

How does a star adjust to angular momentum loss in a stellar wind and how does this depend on the structure of the magnetic field?

We heard from P Charbonneau how an initial fossil field in solar-like stars can lead to the efficient transfer of angular momentum during spin down of stars from angular momentum loss in a stellar wind, and lead to near solid body rotation in the stellar interior as found to be case from helioseismological studies of the solar rotation.

M Pinsonneault reviewed scenarios for angular momentum transport including spin down of young open cluster stars and horizontal branch stars and 
argued that there is still a lot of work to be done to understand how stars evolve with angular momentum transport - a sentiment with which I concur! Understanding the coupling and decoupling between envelope and core remains to be understood.

I Roxburgh stressed that the efficiency of angular momentum loss depends on the topology as well as the magnitude of the large scale magnetic field that controls the angular momentum loss in a stellar wind. The more complex the topology the less is the angular momentum loss since the fraction of the stellar surface that is open to mass loss is less for quadrupole and hexapole fields than for a dipole field and the wind reaches Alfvenic velocities closer into the stellar surface. A Heger pointed out that if the wind is confined to narrow cone $\left(15^{\circ}\right)$ about the rotation axis then stars will spin up.

P Charbonneau and S Sofia and S Barnes emphasised that in the pre-main sequence and early main sequence phases the coupling to a surrounding disc can play a crucial role in determining the angular momentum loss and could explain the spread of rotational velocities in the same cluster. Pinsonneault reminded us that it should be possible to get information on the large scale structure of the magnetic field from X-ray observations.

We have heard of the great efforts made to model mixing processes driven by rotation in stellar evolution. But are we just 'parameter fitting'. Can the effects attributed to rotation equally be explained by convective overshooting?

$\mathrm{S}$ Talon emphasised that the current models of rotational mixing use the least number of parameters and that consistent models are being developed that fit the observations without adjusting parameters. The same view was taken by A Maeder who pointed out that overshooting cannot enhance abundances during main sequence evolution and reminded us of Kippenhahn's remark that the blue loops are like a "magnifying glass" enhancing the effect of different assumptions made about main sequence evolution. Also there are very strong constraints on evolution with different heavy element abundance and these fit into a consistent scenario using rotationally driven mixing.

\section{Conclusions}

In the opinion of the chairman considerable advances have been made, and the importance of magnetic fields in constraining the evolution of the rotation of stars is now recognised. But there is much to be done. In particular to understand the role of a magnetic field in constraining mixing in stellar evolution, on the coupling between different regions of a star, the possibile transfer of angular momentum by oscillations and the effect of chemical composition gradients in supressing mixing processes. This requires both analytical work and numerical simulation which I expect will have been undertaken before the next IAU meeting on Stellar Rotation (and Magnetic Fields).

The upcoming asteroseismology space missions MOST, COROT and Eddington, will provide us with information to probe the interior structure and rotation of stars, and our subject will move from theoretical modelling to an empirically based discipline. We should expect some surprises! 